\title{
Shape and Size Analysis of InAs Nanodots capped by amorphous As by GI-SAXS
}

\author{
K.Kuno ${ }^{1}$, M.Ohtaka ${ }^{1}$, S.Nakano ${ }^{1}$, H.Okuda ${ }^{2}$, S.Ochiai ${ }^{2}$, \\ Y.Noritake ${ }^{3}$, Y.Suzuki ${ }^{3}$, Y.Takeda ${ }^{3}$, and M.Tabuchi ${ }^{4}$ \\ ${ }^{1}$ Graduate Student, Kyoto University, Yoshida,Sakyo-ku,Kyoto 606-8501, \\ ${ }^{2}$ Faculty of Engineering, Kyoto University, \\ ${ }^{3}$ Dept. Mater. Sci. Eng., Nagoya University, ${ }^{4}$ Venture Business Laboratory, Nagoya University, Furo-cho, Nagoya $464-8601$
}

\begin{abstract}
Grazing-incidence small-angle X-ray scattering (GI-SAXS) technique was applied to examine self -assembled InAs quantum dots buried in amorphous arsenic cap layer. The size and the shape assessed from Born approximation of the model structures suggested that the shape of the InAs nanodots grown by molecular beam epitaxy for $2 \mathrm{ML}$ and $4 \mathrm{ML}$ showed a morphological transition from a dome shape into facetted structure.
\end{abstract}

Keywords: quantum dots, GI-SAXS, InAs-GaAs

\section{INTRODUCTION}

Self-assembled semiconducting quantum dots attracted great interest in recent years. The nanodots prepared by self-organization process have been expected for a key material for new electronics and optoelectronics devices [1]. The properties of nanodots are very sensitive to their microstructural parameters, such as their size, shape, spatial distribution and compositions. Therefore we need to evaluate the microstructure in order to use them for devices. The structure of nanodots has been observed by imaging techniques, namely, by using transmission electron microscope (TEM), atom force microscope (AFM), etc [2]. However, scattering techniques have a merit over them that they can assess the microstructure non-destructively. Since nanodots need to be buried in a cap layer for electronic device use, grazing-incidence small-angle scattering (GI-SAXS) has a further merit to examine the microstructure of self-assembled nanodots covered by a cap layer $[3,4]$. In the present study, GI-SAXS results have been analyzed to evaluate the size and the shape of InAs nanodots.

\section{EXPERIMENTAL}

InAs nanodots have been grown on GaAs (001) substrates by molecular beam epitaxy (MBE) [5]-[8]. InAs nanodots layer was grown of 2, 4 and 6 monolayer (ML) thickness at $753 \mathrm{~K}$. In order to apply nanodots to optical divices, InAs nanodots should be capped with a layer having a larger band gap, such as GaAs layer. This in turn causes interdiffusion with nanodots. Therefore this time, we used amorphous arsenic produced at room temperature as protect layer of InAs nanodots against oxidation. GI-SAXS experiments were performed at BL-15A of a synchrotron radiation source, Photon Factory, Tsukuba, Japan. Figure 1 shows present experimental setup. The present measurements were carried out at incident angle of $\theta=0.25^{\circ}$ with the wavelength $\lambda=0.150 \mathrm{~nm}$. A rotation/ translation stage was used to control the X-ray incidence angle and sample height precisely. The in-plane angle, $\phi$, is the angle between the $q_{x}$ direction and [110] direction of the sample. GI-SAXS profiles were taken at $\phi=0^{\circ}$ and $45^{\circ}$ to examine the anisotropy of the shape of nanodots. A CCD detector with a 6-inch Image Intensifier was used for real-time imaging, and then an imaging plate was used for quantitative measurements. The camera length was about $1100 \mathrm{~mm}$.

\section{RESULTS AND DISCUSSION}

Figure 2 shows the two-dimensional GI-SAXS patterns at $\phi=0^{\circ}$ of the samples of $2 \mathrm{ML}$ and $4 \mathrm{ML}$ InAs nanodots layer. The bell-shape pattern observed in $2 \mathrm{ML} \operatorname{InAs}$ sample was derived from 3-dimensional structures of the nanodots. The lower part of the pattern cannot be observed because of the shadow of the sample.

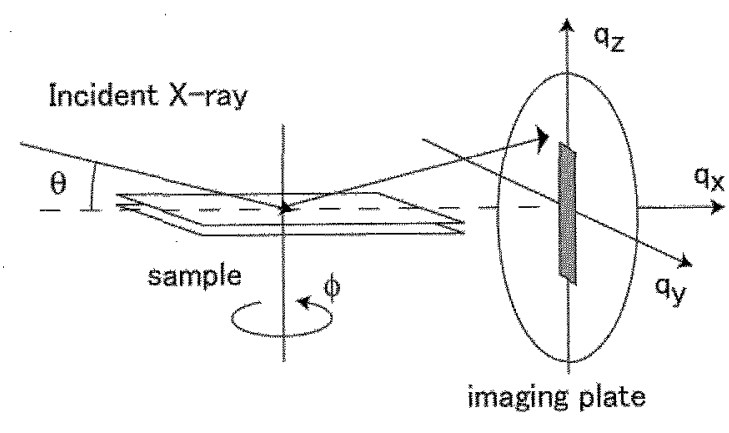

Fig.1 Schematic layout of GI-SAXS experiment 

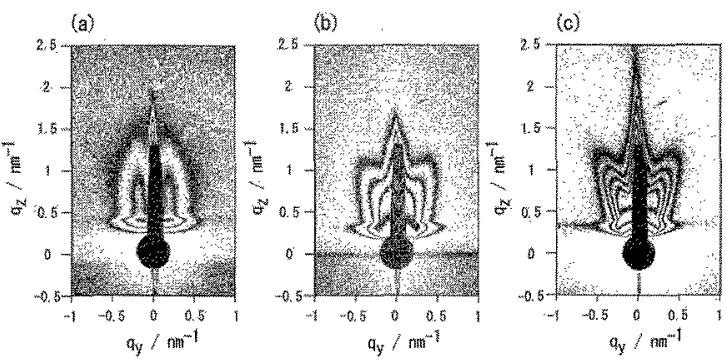

Fig.2 The GI-SAXS patterns from the sample of InAs $2 \mathrm{ML}$ (a), InAs 4ML (b) and $6 \mathrm{ML}(\mathrm{c})$

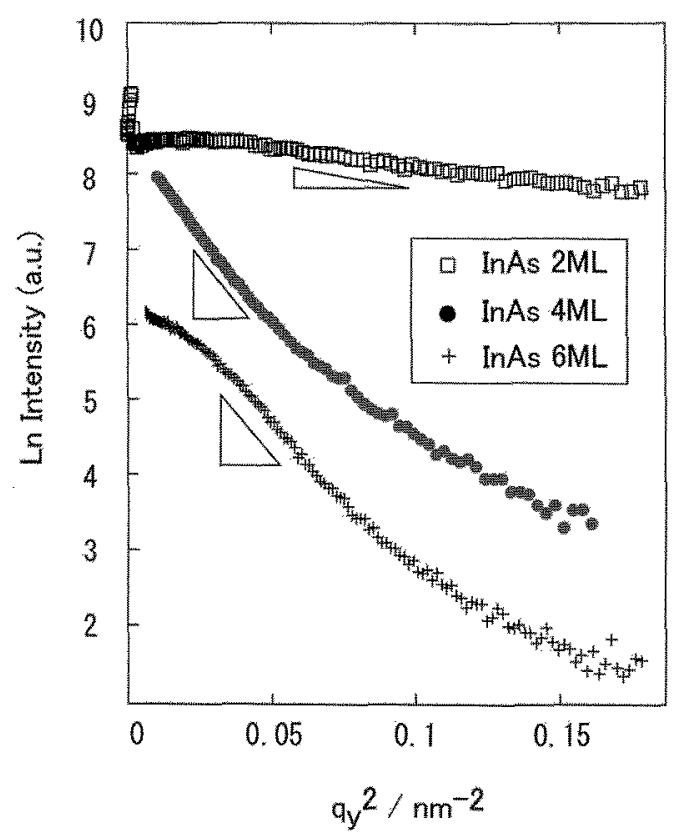

Fig.3 Guinier plots in $\mathrm{q}_{\mathrm{y}}$ direction at $\mathrm{q}_{\mathrm{z}}=0.5 \mathrm{~nm}^{-1} \quad$ (InAs 2ML, 4ML and $6 \mathrm{ML}$ )

In low-q region, scattering intensity was approximated by

$$
\ln (I(q))=\ln (I(0))-\frac{1}{3} R_{g}^{2} q^{2}
$$

where $R_{g}$ is the radius of gyration defined by an average size of the islands

$$
R_{g}^{2}=\frac{\int r^{2} \rho(r) d r}{\int \rho(r) d r}
$$

From Eq.(1), the slope of the $\ln (\mathrm{I}(\mathrm{q}))$ against square of the magnitude of the scattering vector corresponds to the
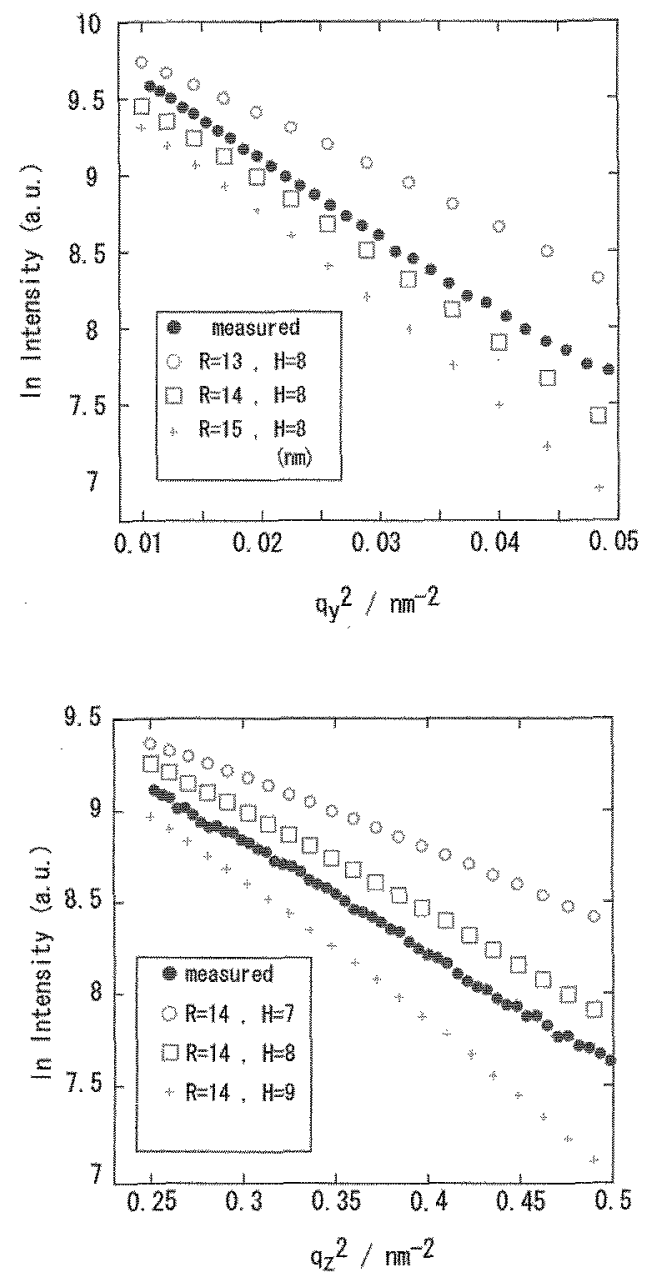

Fig.4 The Guinier plots of measured and simulated pattern. (top : $\mathrm{q}_{\mathrm{y}}$ direction at $\mathrm{q}_{\mathrm{z}}=0.5 \mathrm{~nm}^{-1}$, bottom : $\mathrm{q}_{\mathrm{z}}$ direction at $\mathrm{q}_{\mathrm{y}}=0.15 \mathrm{~mm}^{-1}$ )

average size of the islands. Figure 3 shows Guinier plots of the intensity along $q_{y}$-axis. The Guinier radius in the $q_{y}$ direction should be made at $q_{z}=0$. However the scattering intensity at $\mathrm{q}_{z}=0$ is not accessible and those with the $q_{z}$ close to the Yoneda line should be avoided. Therefore we used the intensity at $\mathrm{q}_{z}=0.5$. The slope of the InAs $4 \mathrm{ML}$ profile is larger than that of InAs $2 \mathrm{ML}$, corresponding to the growth of InAs nanodots. In Fig.2 the streaks suggesting facets were observed for $4 \mathrm{ML}$ InAs, while they were not observed for $2 \mathrm{ML}$. Since relationship between $\mathrm{R}_{\mathrm{g}}$ and the actual size depends on the shape of the dots, we modeled isolated hemisphere-shaped nanodots with various sizes, and simulated the scattering intensities. We determined the actual average size of the InAs nanodots by comparing the Guinier plot of these models and that of measurements.

For a non-faceted nanodot, the outer shape was defined by 


$$
\left(\frac{x}{R}\right)^{2}+\left(\frac{y}{R}\right)^{2}+\left(\frac{z}{H}\right)^{2} \leq 1, z \geq 0
$$

Figure 4 gives Guinier plots of simulated patterns and that of measured GI-SAXS pattern of InAs 4ML sample. The simulated island with radius $\mathrm{R}=14 \mathrm{~nm}$ and height $\mathrm{H}=8 \mathrm{~nm}$ fitted well the measured. In a similar way at InAs 2ML, we estimated radius was $8 \mathrm{~nm}$ and height was 3nm. GI-SAXS data are usually analyzed by the distorted-wave Born approximation (DWBA). However, the approximation is not necessarily valid for the sample with relatively large roughness. Therefore, we adopt Born approximation (BA) and used relatively large $q$ for the analysis.

Since no streaks were observed in the GI-SAXS patterns for InAs 2ML samples, we concluded that $2 \mathrm{ML}$ InAs nanodots had no facet on their surface. As shown in Fig.5 the shapes of the nanodots were assumed to be dome-shape or spheroid.

The streaks observed in the GI-SAXS patterns of the samples 4 and $6 \mathrm{ML}$ InAs suggested that nanodots in these samples are faceted. At in-plane angle $\phi=45^{\circ}$, the streaks were less evident and FWHM of the streaks were wider than these at $\phi=0^{\circ}$, suggesting that the facet on the nanodot was along [110]. The angle between the streak and sample $(x, y)$ base plane in Fig. 2 was about $65^{\circ}$, showing that the streaks are from $\{113\}$-facets. InAs nanodots on GaAs substrate were reported to have $\{113\}$-facets [9]. Therefore we assumed that InAs nanodots have $\{113\}$ facets.

To determine the shape of nanodots, we simulated the scattering intensity from modeled nanodots with, 1. pyramidal structure and 2 . a partly facetted structure defined by the intersection of spheroid and pyramid (Fig.6). The simulated pattern from the absolute pyramid show clear streak, and the angle between the streaks and the substrate plane was similar to the experimental pattern in Fig.2. When facet planes were partial on surface of the dot, the streaks were unclear (Fig.6 bottom). In Fig.2, the streaks were clearer of InAs 6ML than $4 \mathrm{ML}$, so we conclude nanodots were pyramid shapes for InAs 6ML sample, and partly facetted for InAs 4ML sample.

For InAs 4ML sample, we assumed that facetted dot which structure was modeled by an intersection of spheroid and a pyramid. The fraction of the facet changed with growth. Simulation were made for each stage of growth. The size of modeled dot was at spheroid base radius $14 \mathrm{~nm}$ and height $8 \mathrm{~nm}$.

The oscillations of the intensity along qy direction were seen in Fig.6. These oscillations come from form a factor of monodisperse nanodots. To compare the scattering profile with simulation, we assumed that islands have the Gaussian size distribution with $\sigma_{R}=0.2 \vec{R}$, and simulated the scattering patterns for the same form factor with various $R$ (Fig.7). After averaging over size distribution, no oscillation was observed and streaks in relation to $\{113\}$ facet of the island was observed more clearly.

The simulated pattern having $20 \%$ of standard deviation in size agreed fairly well with measured GI-SAXS profiles. It is necessary to analyze and comparing the intensity profile to simulating in Porod region to determine the size distribution quantitatively. However, the statistics is not enough in the present research for a detailed analysis in the Porod region.

For the sample of InAs 6ML, we estimated the size of nanodots in a similar way. The shape was assumed to be perfect pyramid as previously mentioned. The simulated profiles from the pyramidal shape in Fig. 6 agreed with the experimental profiles.

The results obtained by comparing experimental GI-SAXS and simulated profiles are summarized in Table I.

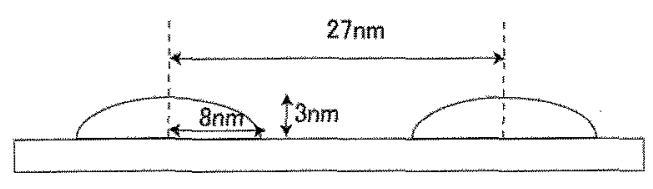

Fig.5 For InAs 2ML, nanodots were dome shape with $\mathrm{R}=8 \mathrm{~nm}$ and $\mathrm{H}=3 \mathrm{~nm}$, and the average interparticle distance $\mathrm{L}$ was $27 \mathrm{~nm}$. L was calculated by $L=2 \pi / q_{y \max }$, where $\mathrm{q}_{\mathrm{ymax}}$ is the peak position of intensity profile along $\mathrm{q}_{\mathrm{y}}$-direction.
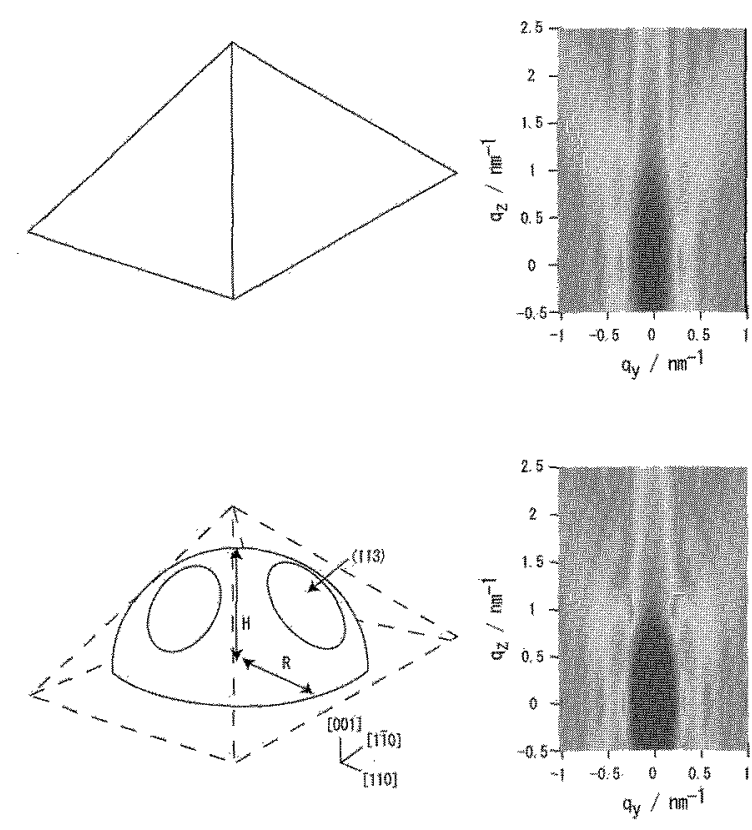

Fig.6 Scattering intensities simulated for the models. The streaks were shown at the similar angle to experimental pattern in Fig.2, and they were clearer for the pyramid shape than for a partly facetted dome. (top : pyramidal shape, bottom $60 \%$ facetted dome), 


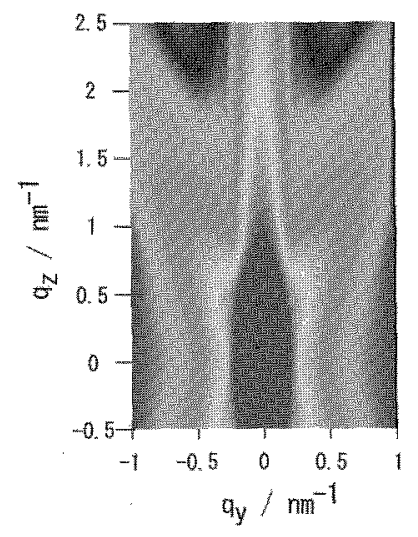

Fig. 7 A GISAXS pattern calculated by BA with a size distribution. Note that the fine oscillations by the form factor are smeared out

table I: size and shape of self-assembled InAs dots

\begin{tabular}{cccc}
\hline sample & radius & height & shape \\
\hline InAs 2ML & $8 \mathrm{~nm}$ & $3 \mathrm{~nm}$ & dome \\
InAs 4ML & $14 \mathrm{~nm}$ & $8 \mathrm{~nm}$ & facetted dome \\
InAs 6ML & $13 \mathrm{~nm}$ & $6.6 \mathrm{~nm}$ & pyramid \\
\hline
\end{tabular}

\section{SUMMARY}

The size and the shape of self-assembled InAs nanodots on GaAs substrate grown by MBE has evaluated by GI-SAXS. With a use of simple BA, we demonstrated that a transition from a dome to pyramid can be assessed. The size of nanodots we estimated increased both in radius and in height as number of InAs layer increased. Therefore the growth of the nanodots was 3-dimensional. We evaluated the shape of the nanodots. The sample of InAs 2ML was dome-shape and InAs 4ML has $\{113\}$-faceted dome shape, which correspond well with the reported process of InAs layer growth on GaAs layer. The facets of the nanodots in InAs $4 \mathrm{ML}$ sample were partial at nanodots surface. Therefore we conclude the shapes of the nanodots were partly $\{113\}$-facetted dome.

\section{REFERENCES}

[1] A. E. Zhokov, A. Y. Egorov, N. A. Maleev and V. M. Ustinov "quantum dot lasers", Oxford University Press, Oxford (2003) pp.63-102

[2] G. Cappellini, L. D. Gaspare, F. Evangelisti and E. Palange : Appl. Phys. Lett. 70, 493-495 (1997)

[3] T. Ogawa, H. Niwa, H. Okuda, S. Ochiai : Materials Science Forum : 475-479, $1097-1100$ (2005)

[4] H. Okuda, S. Ochiai, K. Ito and Y. Amemiya : Appl. Phys. Lett. 81, 2358-2360 (2002)

[5] K. Zhang, Ch. Heyn, W. Hansen, Th. Schmidt and J. Falta : Appl. Phys. Lett. 76, 2229-2231 (2000)

[6] K. Yamaguchi, K. Yujobo and T. Kaizu : Jpn. J. Appl.
Phys. 39, 1245-1248 (2000)

[7] J. C. Gonzalez, F. M. Matinaga, W. N. Rodrigues, M. V. B. Moreira, A. G. de Oliveira, M. I. N da Silva, J. M. C. Vilela, M. S. Andrade, D. Ugarte and P. C. Silva : Appl. Phys. Lett. 76, 3400-3402 (2000)

[8] W. M. McGee, T. J. Krzyzewski and T. S. Jones : J. Appl. Phys. 99, 043505 (2006)

[9] Nabetani et al.: J. Appl. Phys. 76, 347-351 (1994)

(Recieved December 10, 2007 ; Accepted February 26, 2008) 